\title{
ARMADURAS DE COSIDO ENTRE HORMIGONES PREFABRICADOS Y EJECUTADOS IN SITU*
}

\author{
(SEAM REINFORCEMENT BETWEEN TWO CONCRETES PREFABRICATED AND MADE IN SITU)
}

Andrés Solas, Dr. Ingeniero de Caminos

Pontificia Universidad Católica de Chile.

Escuela de Construcción Civil.

\section{RESUMEN}

Este articulo trata sobre el estudio de las armaduras de cosido entre dos hormigones, de distinta naturaleza, que componen un elemento compuesto prefabricado $y$ ejecutado in situ.

La ventaja de este tipo de construcciones es que unidades individuales pueden ser hechas, transportadas y erigidas con mayor facilidad, pudiéndose derivar también algún problema. De todo ello se hace especial referencia a lo largo del articulo.
SUMMARY

This article deals with the study of seam reinforcements between two concretes of different types which make up a compound element prefabricated and made in situ.

The advantage of this type of construction is that the individual units can be made, transported and erected more easily, although some problems may arise. These facts receive special reference throughout the article.

\section{INTRODUCCION}

En este trabajo se estudia el fenómeno de esfuerzo rasante que se produce, a lo largo de la superficie de contacto, entre dos hormigones de distinta naturaleza que componen un elemento compuesto.

Este caso se presenta en la realidad, principalmente en forjados compuestos, construidos generalmente a base de viguetas o placas prefabricadas de hormigón y completadas en obra con una losa superior de hormigón vertido in situ.

El esfuerzo rasante produce en la superficie de contacto de los dos hormigones disímiles o juntas del elemento compuesto, un desplazamiento de un hormigón respecto del otro en el sentido horizontal, consiguiendo con ello que se desarrollen algunos mecanismos

\footnotetext{
* La investigación que se describe, ha sido realizada durante los años 1982 y 1983 en el Laboratorio Central del INSTITUTO TECNICO DE MATERIALES Y CONSTRUCCIONES (INTEMAC) y constituyó la Tesis Doctoral del autor, realizada bajo la dirección del Profesor don José Calavera Ruiz, en la Cátedra de Edificación y Prefabricación de la Escuela Técnica Superior de Ingenieros de Caminos de Madrid.
}

de respuesta como son adherencia y rozamiento entre una cara y otra, en la superficie de contacto; al principio estas dos caras irregulares encajan perfectamente, pero con el corrimiento horizontal estas irregularidades sufren un esfuerzo de corte y, además, tienden a producir un desplazamiento vertical, o lo que algunos autores llaman cabalgamiento, con lo cual activan otro mecanismo tal que las armaduras que atraviesan la superficie de contacto se ponen en tensión por dicho efecto, además de producir el efecto de pasador.

Como la técnica de prefabricación y los prefabricados han sido desarrollados, la construcción de hormigón compuesto ha sido más y más empleada. En este caso, el hormigón prefabricado actuando en conjunción con el hormigón in situ, usualmente de inferior calidad que el prefabricado, forman una sección compuesta terminada.

La principal ventaja de este tipo de construcciones de tipo compuesto, es que unidades individuales pueden ser hechas, transportadas y erigidas con mucha facilidad, incluso el natural monolitismo del vaciado del hormigón in situ es conservado. Aunque se puedan pre- 
sentar algunos problemas en una construcción compuesta como pueden ser:

1. La naturaleza del contacto o unión de la interfase entre los dos hormigones.

2. El efecto de la retracción diferencial en la distribución de tensiones.

3. El efecto de la diferencia de módulo de deformación en la distribución de tensiones.

4. El esfuerzo de tensión y las deformaciones en el hormigón in situ, cuando es limitada o refrenada por la alta calidad del hormigón prefabricado.

5. En general, el comportamiento en carga última.

Esto es en síntesis el enunciado del problema, de acuerdo a lo expresado en los puntos anteriores, es por ello que, en este trabajo, se estudia sólo una parte del problema, la cual se detallará más adelante.

Actualmente el tema de la conexión a esfuerzo rasante está siendo investigado profundamente, especialmente en los paises nórdicos y en un grupo de Trabajo de la Comisión de Prefabricación de la FIP.

Las investigaciones se están orientando principalmente en dos sentidos:
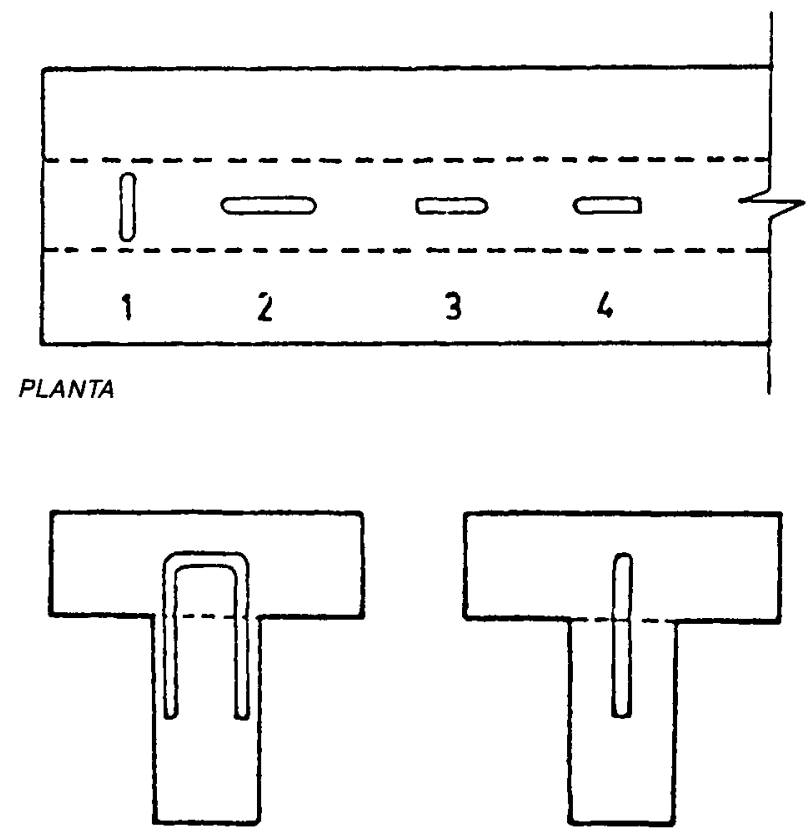

1

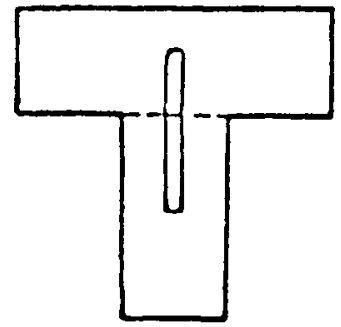

2
1. Valorización de la conexión de los dos hormigones en función de la rugosidad del hormigón prefabricado.

2. Fórmulas para evaluar la contribución a la resistencia de la adherencia entre el hormigón prefabricado y el hormigón in situ y, además, de la armàdura de cosido.

Sinopsis de lo que se pretende con la investigación

Todos los trabajos se basan siempre en la hipótesis de que la armadura transversal de cosido está perfectamente anclada en ambos hormigones.

En la práctica se pueden presentar variadas disposiciones de las armaduras de cosido, algunas de ellas pueden ser como se indica en la figura 1.1.

Estas variadas disposiciones, como las de la figura 1.1., son de eficacia poco conocida, a simple vista dan la impresión de ser muy diferentes unas de otras.

Cuando el espesor $h_{0}$ de la capa hormigonada in situ es pequeño, lo cual es bastante frecuente, las condiciones de anclaje pueden ser deficientes o precarias. Justamente el objeto de esta investigación es el estudio del comportamiento de dichas disposiciones, en una primera etapa, la cual dará como resultado la más
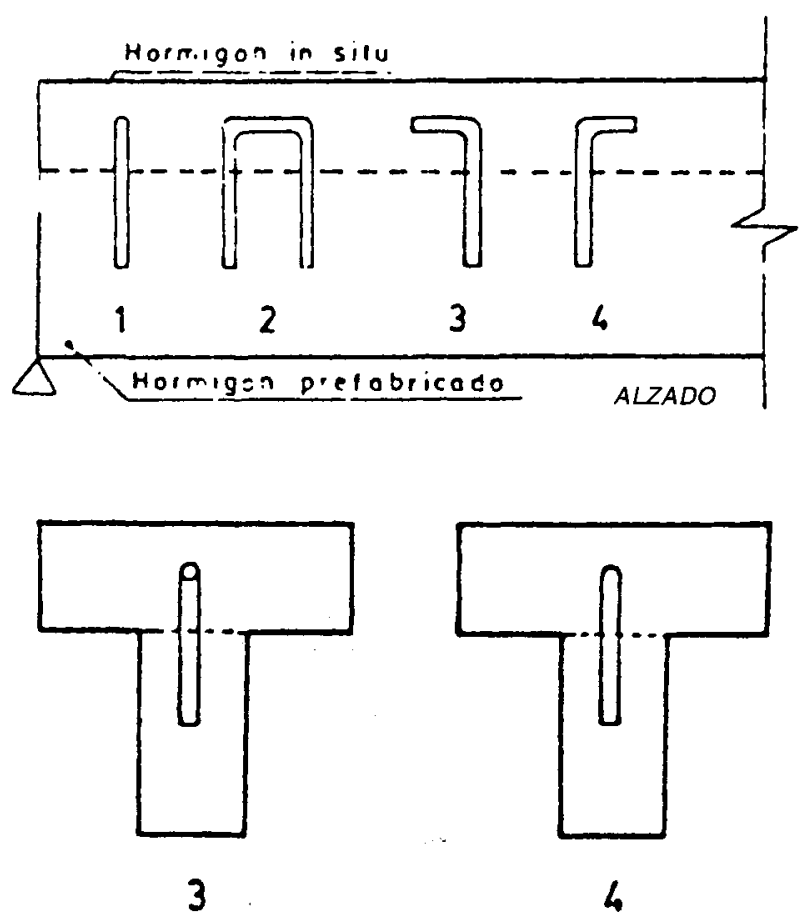

SECCIONES TRANSVERSALES

Fig. 1.1-Disposiciones de armaduras de cosido. 
eficaz contra rasante; una vez determinado lo anterior, esta investigación tenderá a determinar otros parámetros de importancia como son:

a) Forma de las armaduras de cosido, en lo que se refiere al ancho y a la altura de la horquilla y su relación con las condiciones de anclaje.

b) Cuantia de las armaduras de cosido.

\section{MECANISMOS DE TRANSFERENCIA DE CORTANTE}

El corte es transmitido desde un plano a otro por varias vías en elementos de hormigón armado.

La apreciación de algunos de los principales mecanismos de cortante es relativamente reciente y, por eso, la evaluación definitiva de la contribución de ese cortante se ha llevado en componentes, en forma solamente tentativa.

Los principales tipos de transferencia de cortante son:

1. Tensión cortante en el hormigón no fisurado ( $\tau$ us).

2. Transferencia de cortante en la interfase ( $\tau$ us).

Estos conceptos son descritos a continuación:

Tensión cortante en el hormigón no fisurado ( $\tau$ us).

La conexión entre el hormigón in situ y el prefabricado dependen de la resistencia de la junta, ésta depende a su vez de:

a) La adherencia de la superficie de contacto.

b) La rugosidad de la superficie de contacto.

a) La adherencia entre los dos hormigones constituye uno de los parámetros fundamentales en relación con el deslizamiento. De la figura 2.1 se deduce que las juntas adherentes permiten alcanzar valores muy elevados de los esfuerzos rasantes, con una salvedad, que se mantiene muy reducida la magnitud de los correspondientes deslizamientos (no superan los $0,05 \mathrm{~mm}$ ). Esto caracteriza lo que Hanson denomina "uniones rígidas", en comparación con las "uniones dúctiles", que para absorber esfuerzos rasantes importantes es preciso llegar a grandes valores de deslizamientos.

Se ha visto, por ensayos efectuados sobre elementos compuestos, cuya superficie de contacto presentaba buena adherencia, que se comportaron inicialmente du-

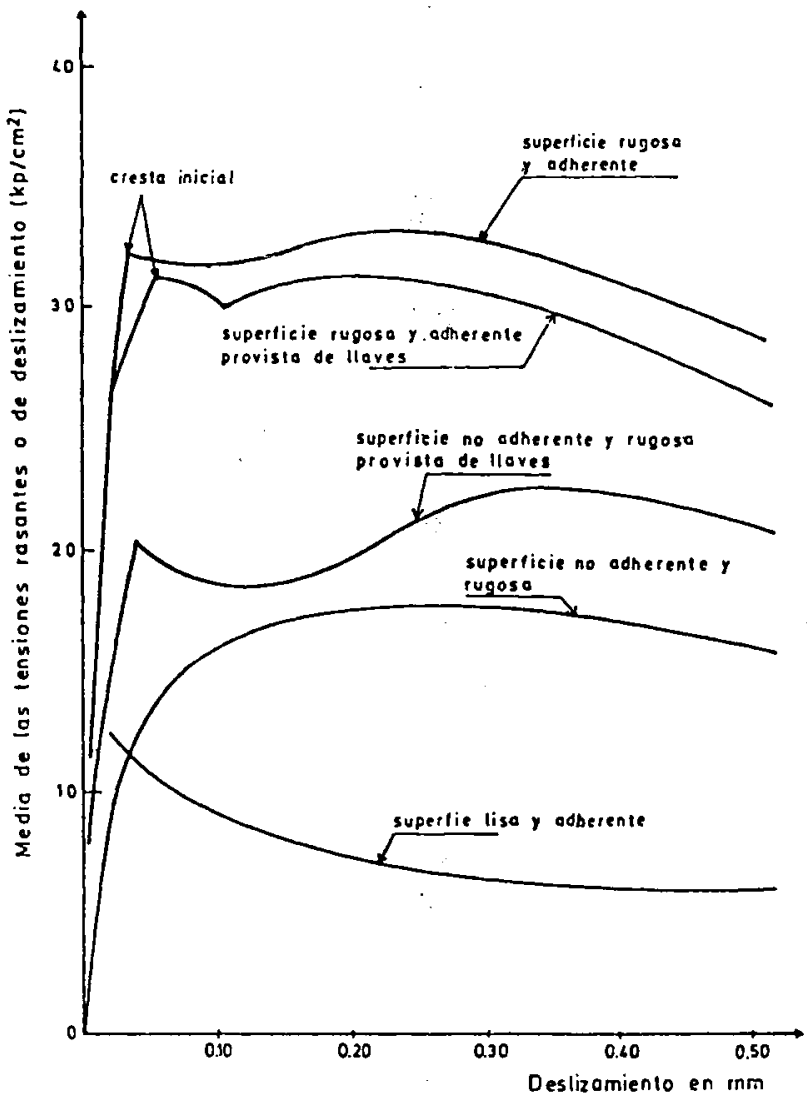

Fig. 2.1.-Influencia del estado de la superficie sobre el valor del deslizamiento.

rante los ensayos en forma análoga a los elementos monolíticos utilizados como testigos.

En consecuencia, al construir estos elementos compuestos debe procurarse conseguir una adherencia lo más perfecta posible. Para ello, la superficie de las piezas prefabricadas debe:

- Estar desprovista de toda suciedad y polvo.

- Procurar evitar al máximo la presencia de lechada en la superficie.

Ensayos realizados por INTEMAC*, referentes al comportamiento de la superficie de contacto entre hormigones vertidos in situ y prefabricados y que se relacionan con el grado de limpieza de la superficie de contacto, han demostrado "que la unión es claramente sensible al polvo interpuesto. La práctica recomendada de limpieza previa debe ser mantenida".

\footnotetext{
* INTEMAC: Instituto Técnico de Materiales y Construcciones (Ma. drid, España).
} 


\section{b) La rugosidad}

La profundidad de las rugosidades superficiales sólo tiene una débil influencia en la capacidad de la junta para absorber los esfuerzos rasantes y que la resistencia de dicha junta depende, fundamentalmente, de que esté más o menos limpia la superficie de contacto.

En ensayos efectuados se ha podido comprobar que el resultado era prácticamente el mismo, tanto en los elementos cuya superficie de contacto se habia hecho rugosa, dejando los áridos al descubierto mediante la eliminación de la lechada superficial, como en aquellos otros en los que dicha superficie era lisa por haber sido terminada con llana.

Otros ensayos han demostrado que la rugosidad proporciona un margen adicional de seguridad frente a la absorción de los esfuerzos rasantes, una vez rota la adherencia. El papel de la rugosidad es análogo al que desempeñan las llaves de hormigón.

Como la profundidad de las irregularidades superficiales no parece tener una importancia primordial con respecto a la resistencia de la superficie de contacto, por ello resulta ilusorio, e incluso desaconsejable, dañar excesivamente la capa superficial del hormigón de la parte prefabricada. Ante todo, es necesario que los gránulos de esta capa estén perfectamente sujetos.

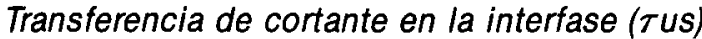

Los diversos investigadores han Ilamado a este mecanismo de muy diversas maneras, casi tantas como investigadores se han dedicado a su estudio; por ejemplo la han Ilamado:

- Transferencia cortante de rugosidad superficial.

- Fricción cortante.

- Transferencia tangencial cortante, etcétera.

El más general puede ser en forma tentativa el de "Rasante en la interfase", que será usado en este trabajo para denotar la fuerza cortante tangencial transmitida a través de un plano de falla.

La teoría de "rasante en la interfase" ha sido discutida en detalle por muchos investigadores, entre los que se pueden citar a Mattock, Binkeland y Mast, etcétera.

En esta teoría se asume que una falla ha ocurrido a lo largo del plano de corte, y teniendo refuerzos de un área total Ast y con un límite elástico de cálculo fyd, que cruzan la falla en ángulo recto. Las caras de la falla serán rugosas e irregulares, asi como cuando el corrimiento ocurre a lo largo de la falla, las piezas de hor- migón a ambos lados de la falla se separan ligeramen. te. Esta separación esforzaria el acero en tracción, lo cual podría volver a producir esfuerzo de compresión en el hormigón que atraviesa a través de la falla. Este esfuerzo de compresión podría proveer una resistencia al deslizamiento a lo largo de la falla, en virtud de la fricción por la rugosidad e irregularidades de las ca. ras de la falla.

Suponiendo que la separación es suficiente para traccionar los esfuerzos hasta el punto de fluencia, de tal modo que si tg $\varnothing$ es el coeficiente de fricción entre las caras de la falla, la fuerza rasante a lo largo de la falla será dado por:

$$
\text { Fs }=\text { Ast fyd } \operatorname{tg} \varnothing
$$

dividiendo por el área del plano de corte, obtenemos la tensión rasante

$$
\tau \text { us }=\text { fyd } \operatorname{tg} \varnothing
$$

Mast propuso que con una rugosidad adherente en la interfase del hormigón prefabricado y el colocado in situ, la tg $\varnothing$ podia tomar valores como 1,4, para valores de fyd no mayores que $0,15 f_{c}^{\prime} \quad\left(f_{c}^{\prime}=\right.$ resistencia a compresión del hormigón).

La teoria de "rasante en la interfase" ignora el efecto de cohesión o de adherencia y lo compensa usando un ángulo aparente de fricción interna, el cual es mu. cho mayor que el real; el ángulo de fricción es sólo "aparente", y es aplicable solamente a niveles bajos de esfuerzos.

En esta investigación se propone una estructura de fórmula diferente para evaluar la tensión rasante de ago. tamiento $(\tau \mathrm{u})$ :

$$
\tau \mathrm{u}=\beta_{1} \tau_{\mathrm{uc}}+\beta_{2} \tau \mathrm{us}
$$

donde:

$\tau$ uc $=$ corresponde al rasante de agotamiento en el hormigón;

$\tau$ us $=$ corresponde al rasante de agotamiento en el acero;

$\beta_{1}$ y $\beta_{2}=$ coeficientes que indican la participación del hormigón y el acero respectivamente.

Esta estructura de fórmula coincide con la del MODEL CODE. 
Además debe cumplirse que:

$$
\tau \mathrm{md} \leq \tau \mathrm{u}
$$

donde:

$\tau \mathrm{md}=$ tensión media rasante de cálculo;

$\tau \mathrm{u} \quad=$ tensión rasante de agotamiento.

Los valores de la tensión media de cálculo $\tau$ md serán de acuerdo al tipo de unión:

\section{Unión dúctil: (Concepto de fuerza rasante)}

Si se da en el caso cuantías importantes de armadura transversal, referida al área de la superficie de contacto $(\varrho$ d). La presencia de la armadura hace dúctil la rotura y permite aceptar una redistribución del fenómeno, al mismo tiempo que reducir los coeficientes de seguridad.

Para que la unión pueda considerarse como dúctil, se debe cumplir la limitación siguiente:

$$
\varrho d \geq \frac{F_{r}}{p L \cdot f y d}
$$

donde:

$\varrho \mathrm{d}=$ cuantia geométrica de la armadura transversal referida al área de la superficie de contacto.

$\mathrm{F}_{\mathrm{r}} \quad$ = Fuerza rasante.

$\mu \quad=$ Coeficiente de rozamiento entre ambos hormigones.

$\mathrm{p} \quad=$ Perímetro de contacto

$\mathrm{L} \quad$ = Luz de la zona correspondiente a la fuerza rasante.

fyd = Límite elástico de cálculo de la armadura transversal. (fyd $\ngtr 4.200 \mathrm{kgf} / \mathrm{cm}^{2}$ ).

La tensión rasante media para unión dúctil se calcula de acuerdo con la siguiente fórmula:

$$
\tau \mathrm{md}=\frac{\mathrm{A}_{\mathrm{s}} \text { fyd }}{\mathrm{pL}} \ngtr \frac{0,85 \text { Ach fcd }}{\mathrm{pL}}
$$

donde:

\footnotetext{
$\mathrm{A}_{\mathrm{s}} \quad=$ Area estricta de armaduras (sección max. momento en vano).
}

Ach = Sección comprimida de hormigón in situ en rotura (máx. momento en vano)

$\mathrm{p} \quad=$ Perimetro de contacto entre hormigón in situ comprimido y pieza prefabricada.

$\mathrm{L}=$ = Longitud de la zona correspondiente.

\section{Unión frágil:}

Es la existente cuando no se dispone de armadura transversal. En este caso la rotura de la adherencia de ambos hormigones se presenta de forma repentina, con un deslizamiento relativo entre ambos sumamente pequeño.

El fenómeno presenta una rotura frágil (sin aviso).

La tensión rasante media $\tau$ md para hormigón frágil se calcula en una sección sometida a flexión (en régimen lineal):

$$
\tau \mathrm{md}=\frac{\mathrm{Vd} S}{\mathrm{pl}}
$$

donde:

Vd = Esfuerzo cortante de cálculo, suponiendo que la sección está en régimen lineal, pero que la pieza ha alcanzado el agotamiento (en general en otra sección).

S = Momento estático de la sección homogeneizada de hormigón in situ respecto al eje OX.

p

$=$ Perímetro de contacto entre los 2 hormigones.

$1 \quad=$ Momento de inercia.

La tensión rasante media para la unión frágil se calcula para grandes momentos flectores (bloque comprimido afecta los dos hormigones) de acuerdo a la siguiente fórmula:

Estados límites últimos

$$
\tau m d=\frac{V d}{p Z}
$$

donde:

Vd $\quad=$ Esfuerzo cortante actuante en la sección.

$\mathrm{Z} \quad=$ Brazo mecánico.

$=$ Perímetro de contacto entre los dos hormigones. 


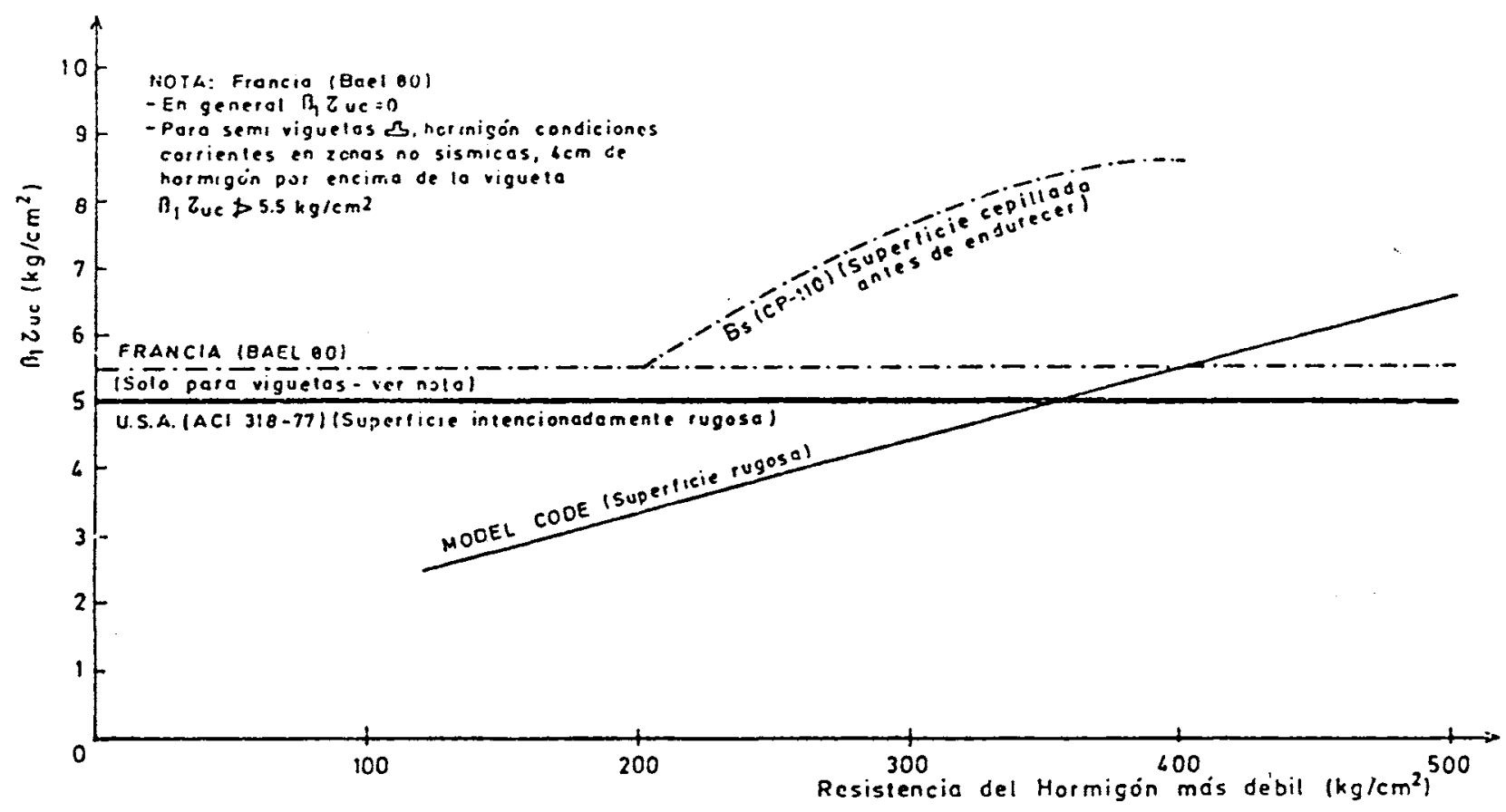

Fig. 2.2.-Comparación de valores $\beta$, y tuc segün las distintas normas.

Por otra parte, queda determinar el valor de $\tau$ u y dicho valor está condicionado por el valor de $\tau$ us, que se de. duce si las armaduras son transversales y perpendiculares a la superficie de contacto con la siguiente fórmula:

$$
\tau \text { us }=\varrho \text { fyd }
$$

donde:

$\tau$ us $\quad=$ Rasante de agotamiento en el acero.

$\varrho \quad=$ Cuantía geométrica de la armadura transversal referida al área de la superficie de contacto.

fyd = Límite elástico de cálculo de la armadura transversal.

Por otro lado, el valor de $\tau$ u también está condicionado al valor de $\tau$ uc; las discrepancias de unas Normas a otras es importante y no puede serlo por menos, ya que el valor $\tau$ uc está fuertemente ligado a la rugosidad y al estado de la superficie del hormigón de la pieza prefabricada, tal como se discutió con anterioridad a título de ejemplo; en la figura 2.2 se indican los valores adoptados de $\beta_{1} \tau$ uc por el $\mathrm{ACl} 318$, por la Norma Británica CP-110 y por la Norma Francesa Bael-80.
En esta investigación se han encontrado algunos valores de contribución del hormigón $\tau$ uc a esfuerzo rasante, así como también se han podido evaluar algunos valores de los coeficientes $\beta_{1}$ y $\beta_{2}$ y que se presentarán más adelante en resumen de resultados, $c 0$. mo también en la parte de conclusiones.

Los valores de $\tau$ US, $\tau$ uc, $\beta_{1}$ y $\beta_{2}$ que son experimentalmente obtenidos a través de esta investigación, permiten determinar con bastante exactitud el valor de $\tau$ u (tensión rasante de agotamiento) que se comparará como se dijo anteriormente con la $\tau$ md (tensión media de cálculo).

\section{INVESTIGACION EXPERIMENTAL EN LABORATORIO}

\subsection{Juntas con armaduras de cosido}

Estos ensayos tienen como finalidad estudiar la influencia de la disposición, forma y cuantía de las armaduras de cosido en la contribución al esfuerzo rasante, en cada caso.

\subsection{Primera fase de la investigación (Serie II-A)}

En esta fase de la investigación se realizan ensayos para determinar cuál es la disposición óptima de armaduras de cosido que permiten absorber la mayor cantidad de esfuerzo rasante. 


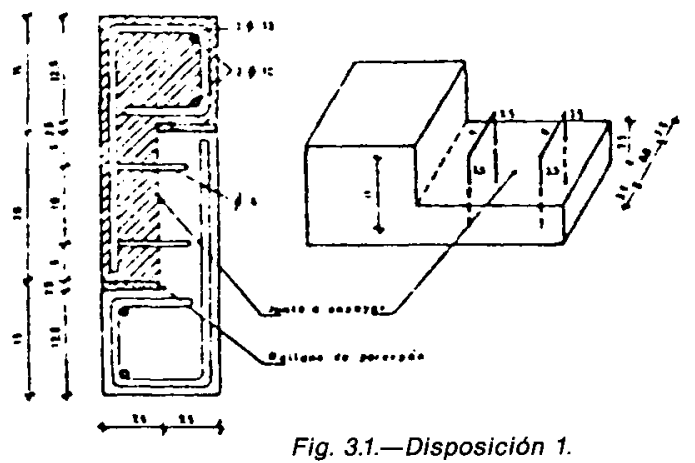

Fig. 3.1.-Disposicion 1.

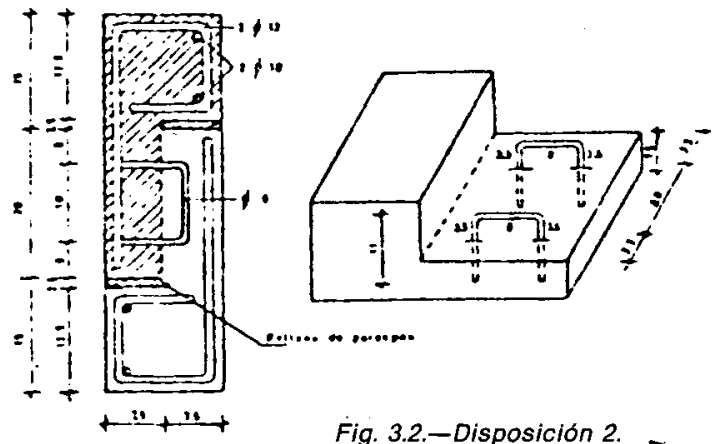

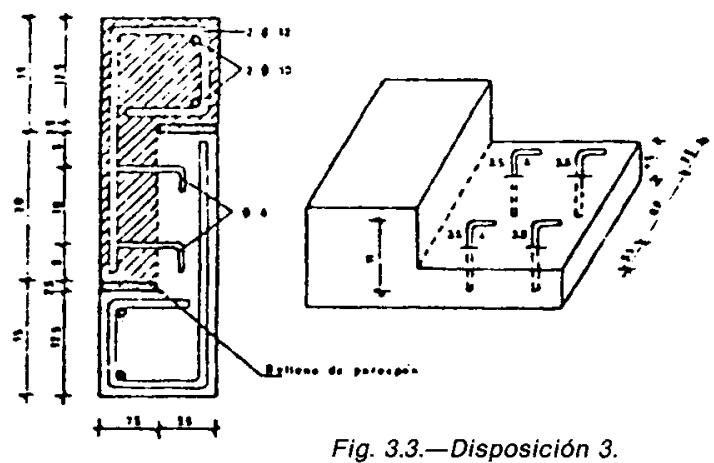

Fig. 3.3.-Disposición 3.

NOTA: La zona rayada es $\mathrm{H}-350$ hormigón prefabricado. La zona sin rayar es $\mathrm{H}-200$ hormigón in situ.

\subsubsection{Disposición de las armaduras de cosido}

Las disposiciones de las armaduras de cosido analizadas son cuatro:

\section{Disposición 1:}

Estribo cerrado en el hormigón in situ, con su plano ortogonal al esfuerzo rasante.

\section{Disposición 2:}

Estribo cerrado en el hormigón in situ, con su plano paralelo al esfuerzo rasante.

\section{Disposición 3:}

Horquilla abierta (mitad del estribo de disposición 1 y 2) en el hormigón in situ, con su medio plano paralelo al esfuerzo rasante y con dirección de la abertura de la horquilla opuesta a la del esfuerzo rasante.

\section{Disposición 4:}

Horquilla abierta (mitad del estribo de la disposición 1 y 2) en el hormigón in situ, con su medio plano paralelo al esfuerzo rasante y con dirección de la abertura de horquilla igual a la del esfuerzo rasante.

\subsubsection{Descripción de las probetas y sus armaduras}

Se emplean probetas de $20 \mathrm{~cm}$ de longitud en la junta y $15 \mathrm{~cm}$ de ancho. El hormigón prefabricado tiene 7,5 centímetros de espesor, así como la losa in situ. Las piezas llevan las cabezas descritas en las figuras 3.1 a 3.4, para transmitir por compresión los esfuerzos de corte.

Las disposiciones de las armaduras son las señaladas esquemáticamente en las figuras 3.1 a 3.4 y son de diámetro $6 \mathrm{~mm}$ (AEH-500), y tienen suficiente longitud de anclaje. Además se conserva constante la cuantía de armaduras, en las cuatro disposiciones. Se muestra en la figura 3.5 el detalle de la armadura de cosido y sus dimensiones.

\subsubsection{Características de los hormigones prefabricados in situ}

Hormigón prefabricado: Se realizó con hormigón tipo $\mathrm{H}-350$ y se ajustó la cantidad de agua para obtener un asiento de cono de $5 \pm 1 \mathrm{~cm}$. Este hormigón se vierte en una capa de $7,5 \mathrm{~cm}$ de espesor, se compacta con vibrador de aguja de una frecuencia de 12.000 r.p.m. y 


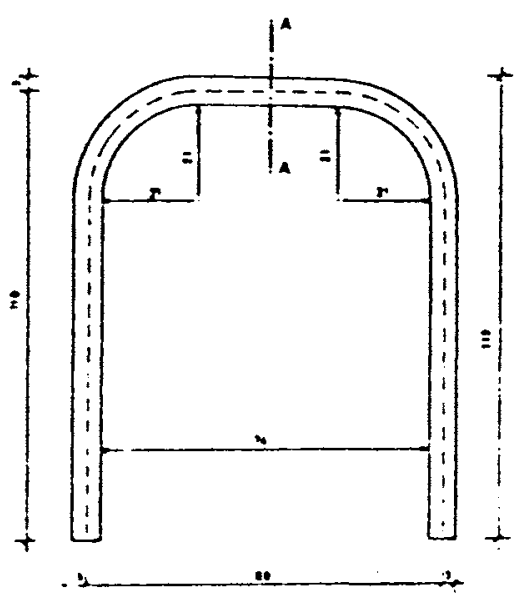

NOTA: Las armaduras de las disposiciones 3 y 4 se obtuvieron cortando en A-A, el estribo representado arriba.

Fig. 3.5.-Juntas serie II-A. Armadura de cosido.

de $25 \mathrm{~mm}$ de diámetro, y posteriormente se maestreo con regla de madera. El curado se realizó en ambiente a $20{ }^{\circ} \mathrm{C}$ y $60 \%$ de H.R.

La dosificación por metro cúbico usada en este hormigón es:

Grava de machaqueo $(20 \mathrm{~mm})$

Arena rodada $(2 \mathrm{~mm})$

Cemento Portland P.450

Agua

$1.300 \mathrm{~kg}$

$650 \mathrm{~kg}$

$325 \mathrm{~kg}$

$175 l$

Las resistencias a compresión se determinan con 4 probetas cilindricas de $15 \times 30 \mathrm{~cm}$, curadas en las condiciones de las piezas. Los resultados de los ensayos del hormigón H-350 empleados son los siguientes:

- Resistencia promedio a 7 días

$310 \mathrm{kgf} / \mathrm{cm}^{2}$

- Resistencia promedio a 28 dias

$358 \mathrm{kgf} / \mathrm{cm}^{2}$

Hormigón in situ: Se hizo con hormigón tipo H-200 y con un asiento de $5 \pm 1 \mathrm{~cm}$. Este hormigón se coloca en una capa de $7,5 \mathrm{~cm}$ de espesor. El procedimiento de vibrado y curado es análogo al del hormigón prefabricado. Este hormigón in situ se vierte cuando el hormigón prefabricado $(\mathrm{H}-350)$ tiene al menos 7 días de edad.

La dosificación por metro cúbico es de:

Grava rodada $(20 \mathrm{~mm})$

Arena rodada $(2 \mathrm{~mm})$

Cemento Portland P-350

Agua

$1.300 \mathrm{~kg}$

$650 \mathrm{~kg}$

$325 \mathrm{~kg}$

$175 l$
Las resistencias a compresión se determinan con 4 probetas cilíndricas de $15 \times 30 \mathrm{~cm}$, curadas en las condiciones de las piezas y los resultados de ensayo del hormigón $\mathrm{H}-200$ son:

- Resistencia promedio a 7 dias

$177 \mathrm{kgf} / \mathrm{cm}^{2}$

- Resistencia promedio a 28 días $238 \mathrm{kgf} / \mathrm{cm}^{2}$

La edad de este hormigón, en el momento de ensayar las juntas de las piezas, es de aproximadamente 28 días.

\subsubsection{Acero de las armaduras de cosido}

Las armaduras de cosido empleadas $(\varnothing 6 \mathrm{~mm}$ ) en la serie II-A son de acero AEH $500 \mathrm{~N}$ con las siguientes características medias:

- Límite elástico (fy)

$=5.720 \mathrm{kgf} / \mathrm{cm}^{2}$

- Carga unitaria de rotura (fs) $=6.900 \mathrm{~kg} / \mathrm{cm}^{2}$

- Alargamiento de rotura $(S / 5 \varnothing)=18 \%$

En la serie II-C se empleó acero AEH $400 \mathrm{~N}$ de diámetro $6 \mathrm{~mm}$ en las armaduras de cosido. Las caracteristicas fundamentales son las siguientes:

$\begin{array}{llr}\text { - Limite elástico (fy) } & =4.320 \mathrm{kgf} / \mathrm{cm}^{2} \\ \text { - Carga unitaria de rotura (fs) } & =6.720 \mathrm{kgf} / \mathrm{cm}^{2} \\ \text { - Alargamiento de rotura }(S / 5 \varnothing) & = & 32 \%\end{array}$

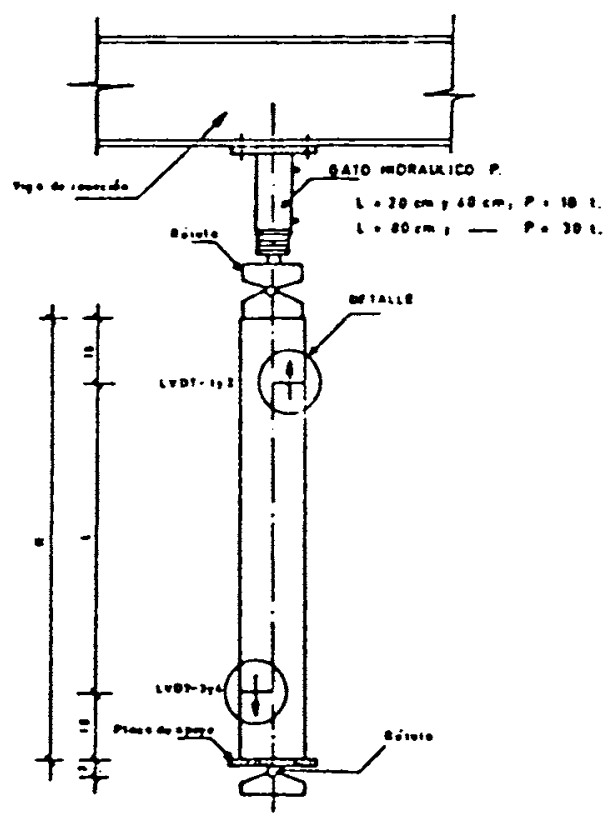

Fig. 3.6.-Montaje de los ensayos. http://informesdelaconstruccion.revistas.csic.es 


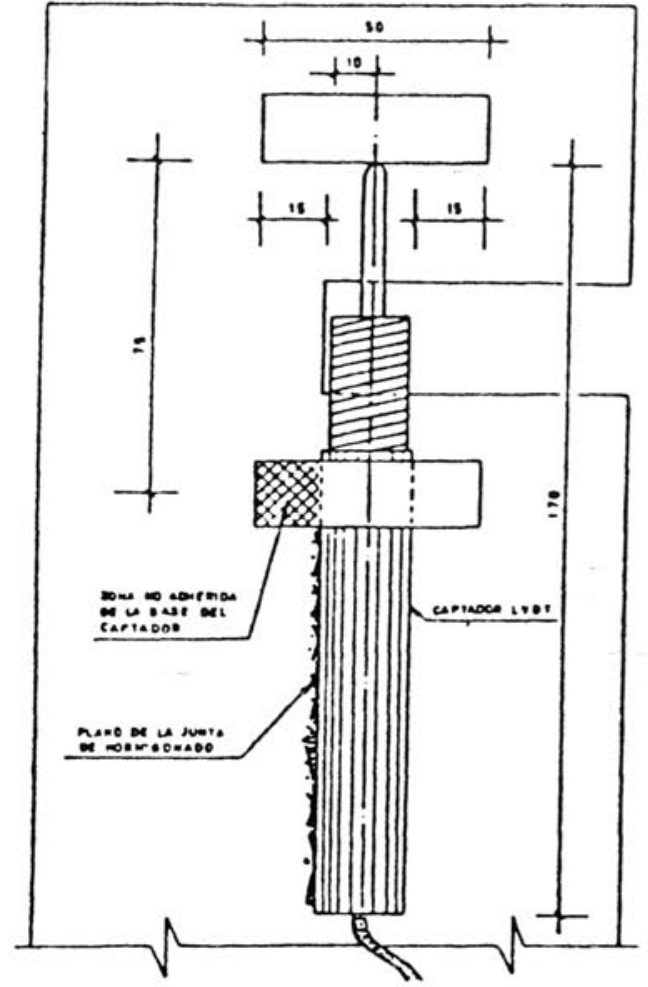

Fig. 3.7.-Detalle (Fig. 3.6).

\subsubsection{Montaje de los ensayos}

El montaje de los ensayos es idéntico en la serie II-A y II-C.

Los ensayos se montaron de acuerdo a las figuras 3.6 y 3.7 y fotografía 1, para ello se empleó un gato K.W. de 10 ó 30 toneladas (según el largo de la junta) suspendido de una viga de reacción y apoyado sobre una rótula centrada con el plano de la junta de la pieza; dicha pieza a su vez estaba apoyada en otra rótula que descansaba sobre el suelo.

Para medir los desplazamientos, se dispusieron captadores inductivos en cada extremo de la probeta, éstos estaban conectados a un ordenador, asi como los captadores de carga.

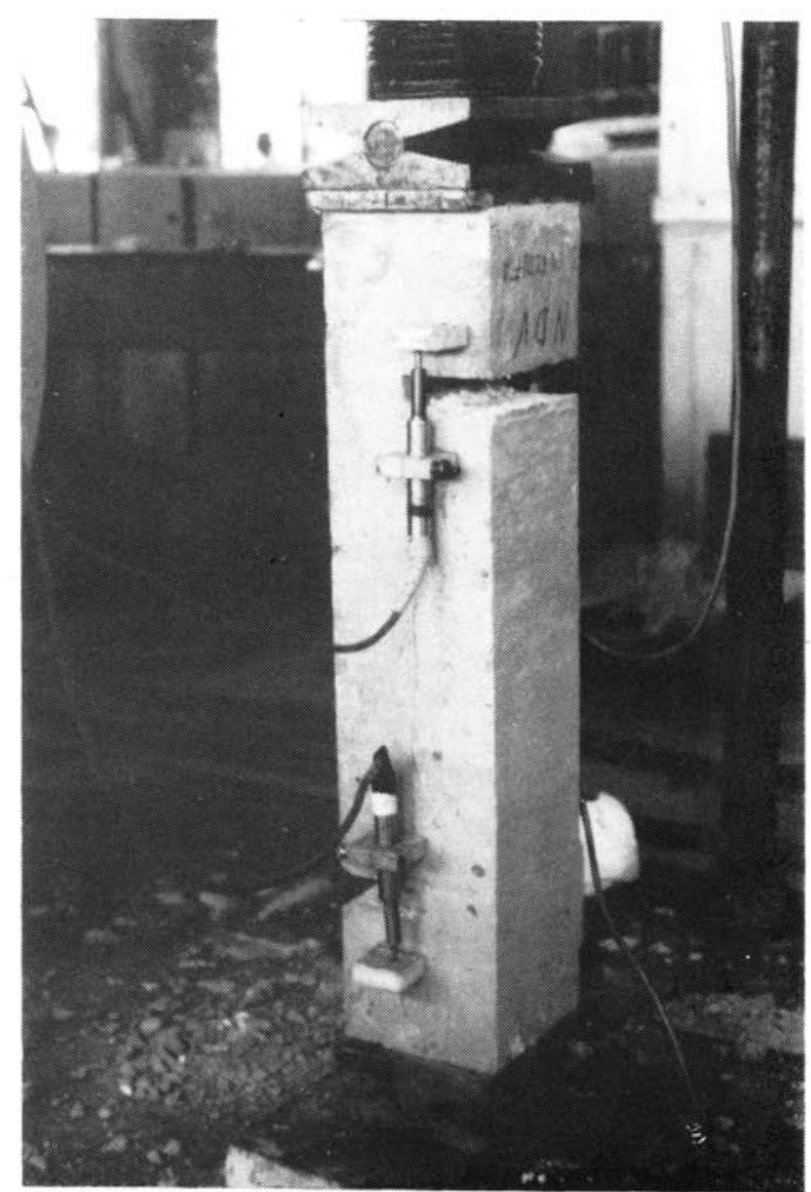

Foto 1.-Serie I. Montaje del ensayo.

\subsubsection{Resumen de resultados de la primera fase (Serie II-A)}

Se incluyen aquí los resultados de los ensayos de las probetas de la serie II-A, de acuerdo a las cuatro disposiciones en estudio.

La cuantía de las armaduras de cosido en esta serie es de $3,77 \times 10^{-3}$.

Tabla 3.1.-Resumen de resultados

\begin{tabular}{|cc|cc|cc|cc|}
\hline \multicolumn{2}{|c|}{ Disposición 1 } & \multicolumn{2}{c|}{ Disposición 2 } & \multicolumn{2}{c|}{ Disposición 3 } & \multicolumn{2}{c|}{ Disposición 4 } \\
\hline $\begin{array}{c}\tau u \\
\mathrm{kgf} / \mathrm{cm}^{2}\end{array}$ & $\begin{array}{c}\delta \mathrm{u} \\
\mathrm{mm}\end{array}$ & $\begin{array}{c}\tau \mathrm{u} \\
\mathrm{kgf/cm}\end{array}$ & $\begin{array}{c}\delta \mathrm{u} \\
\mathrm{mm}\end{array}$ & $\begin{array}{c}\tau \mathrm{u} \\
\mathrm{kgf/cm} \mathrm{cm}^{2}\end{array}$ & $\begin{array}{c}\delta \mathrm{u} \\
\mathrm{mm}\end{array}$ & $\begin{array}{c}\tau \mathrm{u} \\
\mathrm{kgf/} / \mathrm{cm}^{2}\end{array}$ & $\begin{array}{c}\delta \mathrm{u} \\
\mathrm{mm}\end{array}$ \\
\hline 35,1 & 0,655 & 26,2 & 0,570 & 25,5 & 0,396 & 26,6 & 0,445 \\
\hline
\end{tabular}

$\tau \mathrm{u}=$ Tensión tangencial media.

$\delta \mathrm{u}=$ Deslizamiento medio de los extremos de la junta bajo carga máxima. 


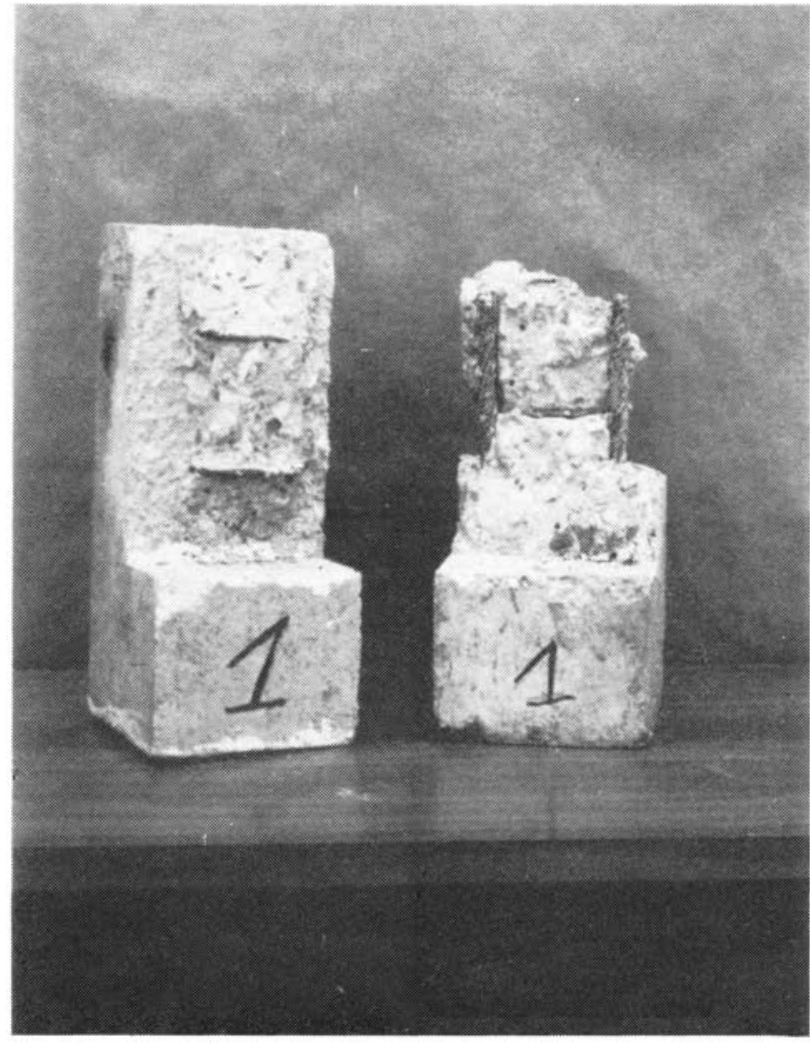

Foto 2.-Serie II-A. Forma de rotura de la pieza con las armaduras de conexión (tipo 1).

Las fotografias 2 y 3 muestran las formas de rotura obtenidas con las armaduras de cosido tipo 1 y 3.

\subsubsection{Análisis de los resultados de la serie II-A}

Se puede apreciar en la tabla Resumen de Resultados de la serie II-A, que éstos conducen a considerar óptima la designada "Disposición 1" con armaduras de cosido, formada por estribo cerrado en el hormigón in situ, con su plano ortogonal al esfuerzo rasante y con sus extremos debidamente anclados en el hormigón prefabricado. Esta "Disposición 1" es la elegida para continuar con la serie II-C, haciendo variar el ancho y la altura de los estribos, así como su cuantia.

\subsection{Segunda fase de la investigación (Serie II.C)}

En esta fase de la investigación se realizan ensayos para determinar cómo influyen la forma y cuantía de las armaduras de cosido, con "Disposición 1", en la contribución al esfuerzo rasante, en cada caso. La "forma" de las armaduras de cosido implica el cambio de dos variables en los estribos cerrados con "Disposición 1" y éstas son: variación en el ancho y en la altura; esta (c) Consejo Superior de Investigaciones Científicas Licencia Creative Commons 3.0 España (by-nc)

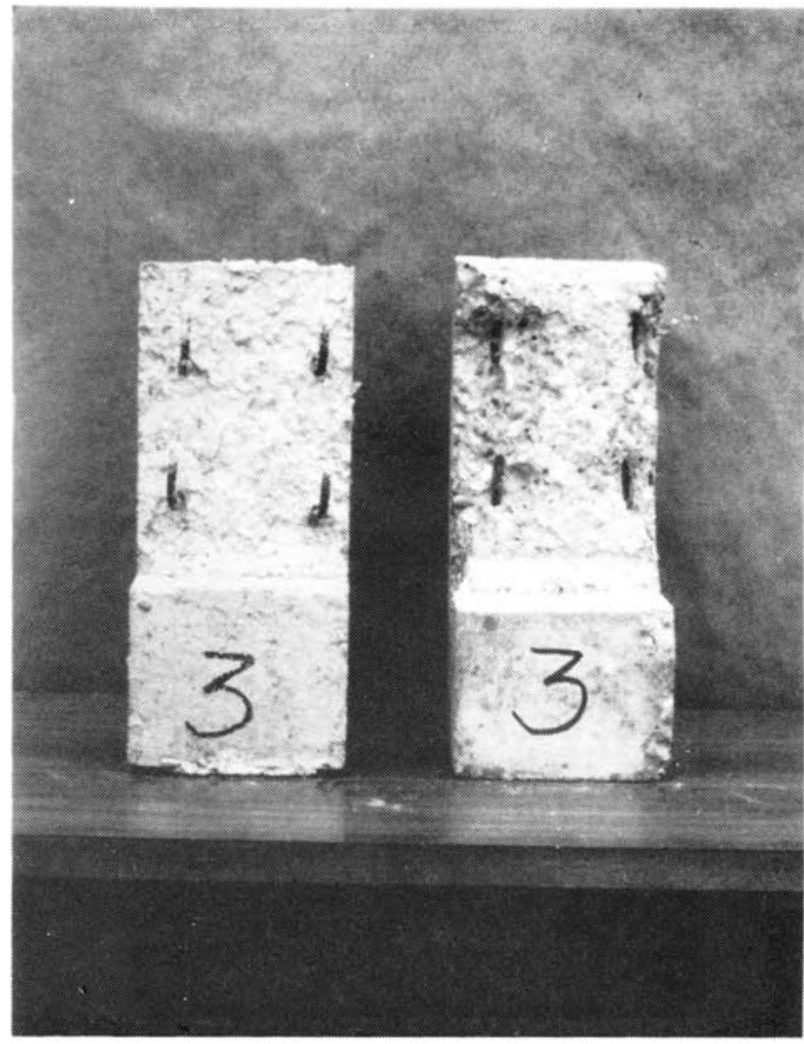

Foto 3.-Serie II-A. Forma de rotura de la pieza con las armaduras de conexión (tipo 3).

última permite investigar, además, sobre la variación de recubrimiento y longitud de anclaje.

\section{Cuantías}

Alta: Igual a la de la serie II-A

$$
\left(\varrho=3,77 \cdot 10^{-3}\right) \text {. }
$$

Media: Mitad de la serie II-A

$$
\left(\varrho=1,88 \cdot 10^{-3}\right) .
$$

Baja: Cuarta parte de la serie II-A

$$
\left(\varrho=0,94 \cdot 10^{-3}\right) \text {. }
$$

En resumen, se tienen 12 posibilidades de variación de los ensayos igual a 2 anchos de estribos por 2 alturas de estribos y por 3 cuantias.

\subsubsection{Forma y cuantía de las armaduras de cosido}

Forma implica: a) Ancho del estribo

b) Altura del estribo

http://informesdelaconstruccion.revistas.csic.es 


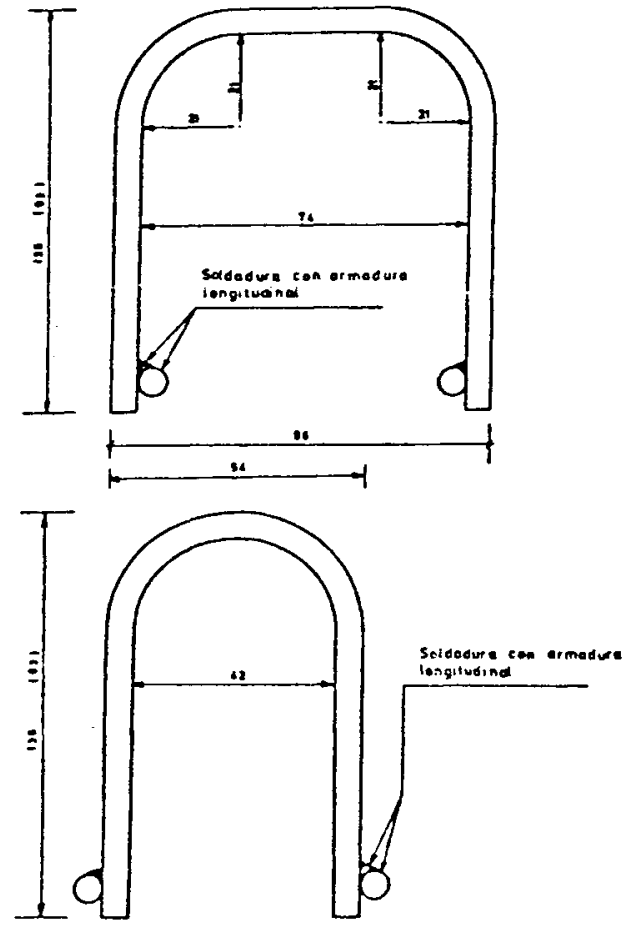

Fig. 3.8.-Juntas serie II-C. Armadura de cosido $\varnothing 6$.

a) Ancho del estribo:

1. Es el mínimo constructivo con $\varnothing 6$ ancho mínimo de $54 \mathrm{~mm}$ (Fig. 3.8).

2. Es el mismo que en la serie II-A de $86 \mathrm{~mm}$. (Figura 3.8).

b) Altura del estribo (Fig. 3.8):

1. Altura menor: A $2 \varnothing$ de la cara inferior de la losa in situ.

2. Altura mayor: A $15 \mathrm{~mm}$ de la cara superior de la losa in situ.

\subsubsection{Descripción esquemática de las probetas y sus armaduras}

Se emplean en esta serie II-C probetas, con el doble de superficie de junta que en la serie anterior, o sea, $40 \mathrm{~cm}$ de longitud de la junta y $15 \mathrm{~cm}$ de ancho.

El hormigón prefabricado tiene $7,5 \mathrm{~cm}$ de altura y análogamente la losa in situ. Las piezas llevan las cabezas descritas en las figuras $3.9,3.10$ y 3.11 , para transmitir por compresión los esfuerzos de corte.

La forma y cuantía de las armaduras se señalan con mayor detalle, de forma esquemática, en las figuras 3.9, 3.10 y 3.11 y en las fotografías 4,5 y 6 . Estas armaduras son de diámetro $6 \mathrm{~mm}$ AEH-400N; se disponen soldadas a la armadura longitudinal del hormigon prefabricado, mejorando su anclaje en éste.

(c) Consejo Superior de Investigaciones Científicas

Licencia Creative Commons 3.0 España (by-nc)

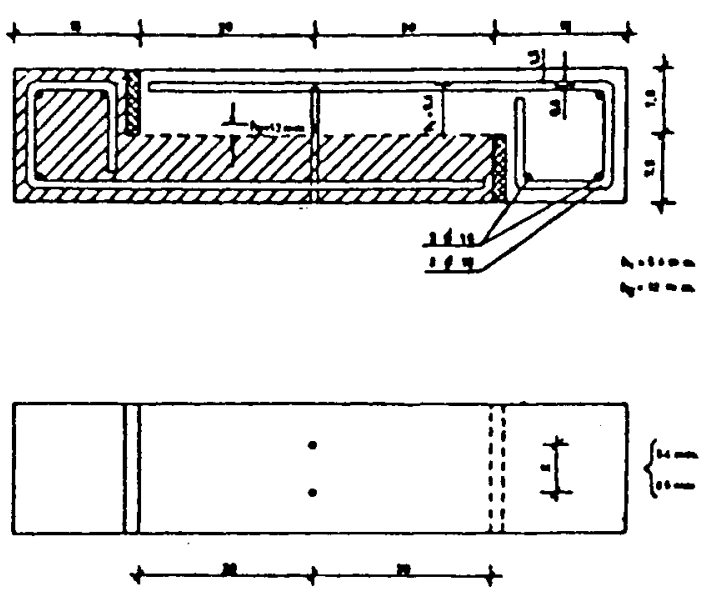

Fig. 3.9.-Esquema de armadura para cuantía baja.
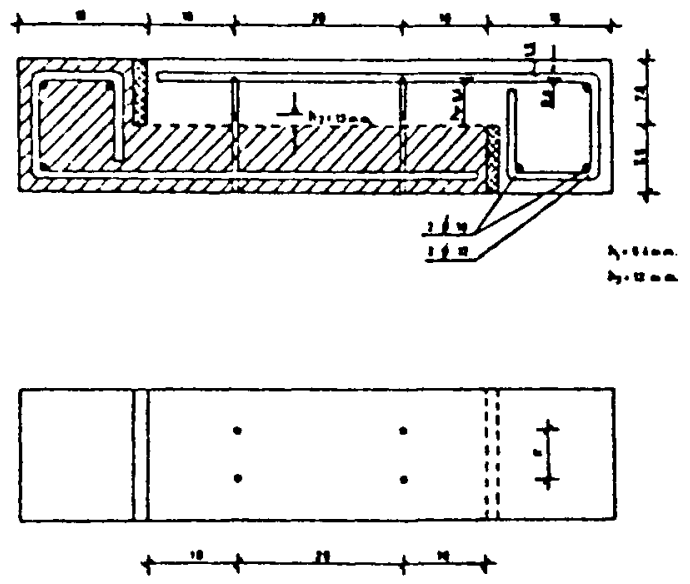

Fig. 3.10.-Esquema de armadura para cuantia media
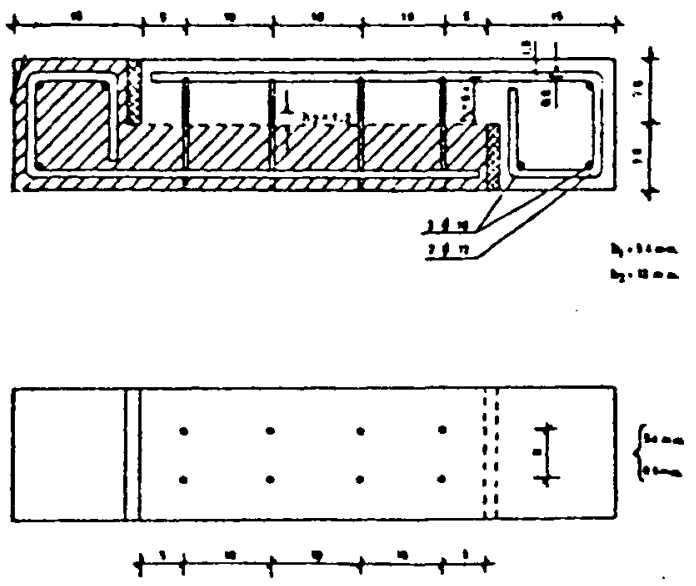

Fig. 3.11.-Esquema de armadura para cuantia alta. http://informesdelaconstruccion.revistas.csic.es 


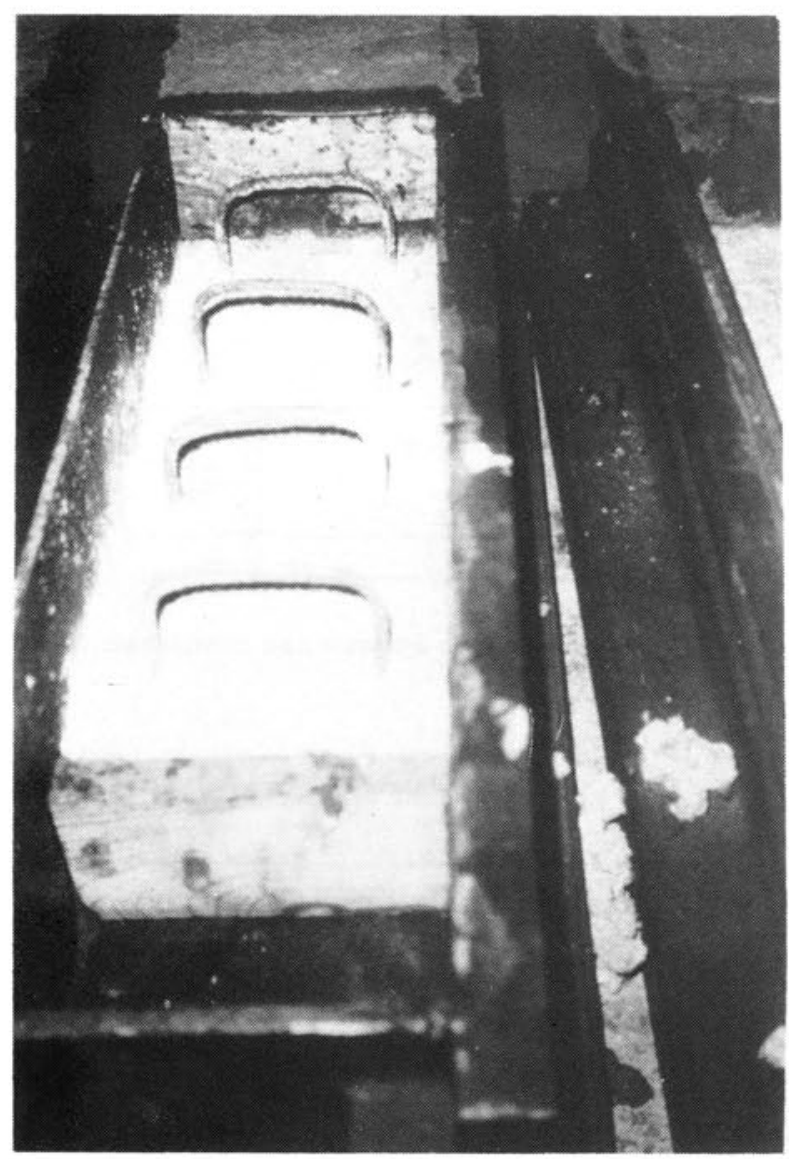

Foto 4.-Serie II-C. Cuantía alta. Pieza tipo 1.

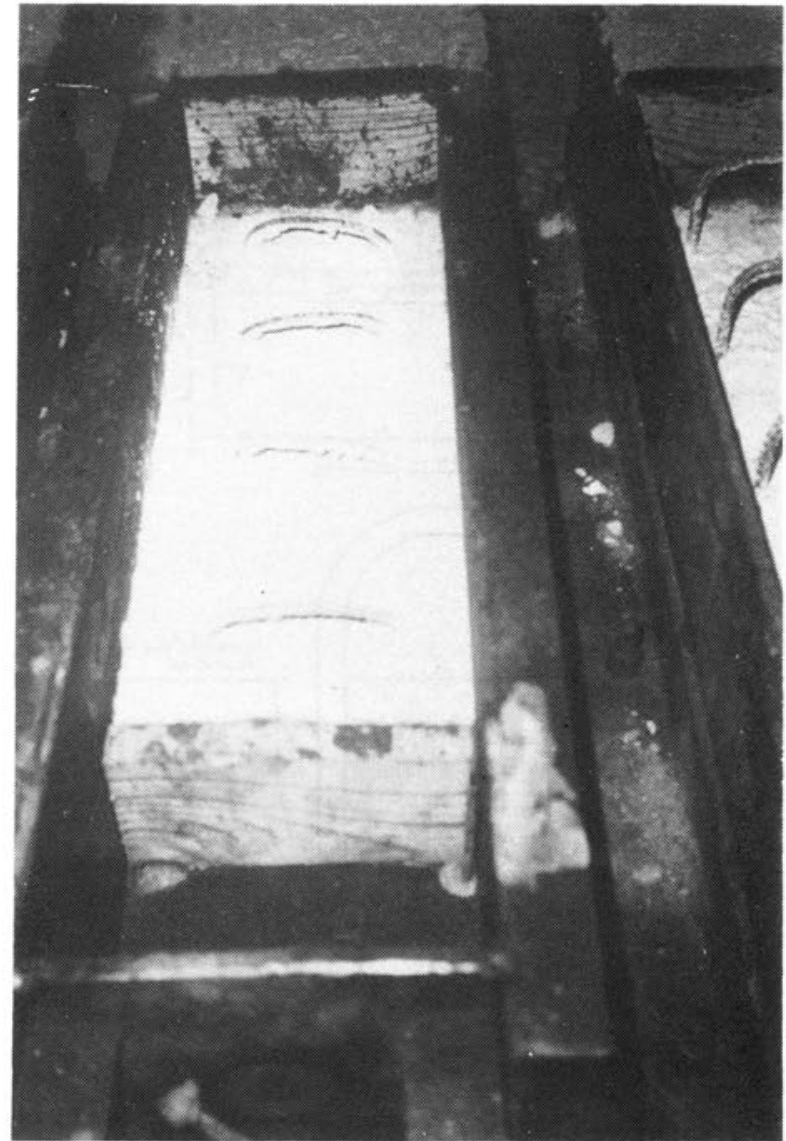

Foto 5.-Serie II.C. Cuantia alta. Pieza tipo 2.

Tabla 3.2.-Resumen de resultados

\begin{tabular}{|c|c|c|c|c|c|c|c|c|c|c|c|}
\hline \multicolumn{4}{|c|}{ ESOUEMA } & & & & & & & & \\
\hline \multirow{2}{*}{\multicolumn{3}{|c|}{ PIEZA TIPO }} & \multirow{2}{*}{$\stackrel{\bullet}{\vdots}$} & \multicolumn{2}{|c|}{1} & \multicolumn{2}{|c|}{2} & \multicolumn{2}{|c|}{3} & \multicolumn{2}{|c|}{6} \\
\hline & & & & $z_{0}$ & $\mathbf{S}_{\mathbf{v}}$ & $z_{0}$ & $\boldsymbol{J}_{.}$ & $z_{0}$ & $J_{.}$ & $z_{u}$ & $J_{u}$ \\
\hline \multirow{6}{*}{$\begin{array}{l}u \\
= \\
w \\
\bar{\alpha} \\
w \\
\sim\end{array}$} & \multirow{6}{*}{ 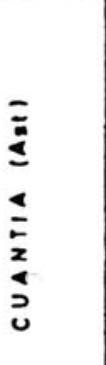 } & \multirow[t]{2}{*}{ B } & \multirow{2}{*}{$\begin{array}{l}a \\
b \\
m\end{array}$} & \multirow[t]{2}{*}{11.38} & $\begin{array}{l}0.210 \\
0.509\end{array}$ & \multirow[t]{2}{*}{0.10} & $\begin{array}{l}0221 \\
0286 \\
\end{array}$ & \multirow[t]{2}{*}{10.86} & $\begin{array}{l}0.553 \\
1.076\end{array}$ & \multirow[t]{2}{*}{231} & $\begin{array}{l}0.126 \\
0.172\end{array}$ \\
\hline & & & & & 2,486 & & 204 & & 0.016 & & edse \\
\hline & & \multirow[t]{2}{*}{ M } & \multirow{2}{*}{$\begin{array}{l}0 \\
b \\
m\end{array}$} & \multirow[t]{2}{*}{18.01} & $\begin{array}{l}0.001 \\
2.671\end{array}$ & \multirow[t]{2}{*}{11.91} & $\begin{array}{l}0.167 \\
0.118\end{array}$ & \multirow[t]{2}{*}{18.53} & $\begin{array}{l}0.500 \\
0.532\end{array}$ & \multirow[t]{2}{*}{1296} & $\begin{array}{l}0.306 \\
0.165\end{array}$ \\
\hline & & & & & 291 & & 2,160 & & 0.66 & & 0.110 \\
\hline & & \multirow[t]{2}{*}{ A } & \multirow{2}{*}{$\begin{array}{l}a \\
b \\
m\end{array}$} & \multirow[t]{2}{*}{22.06} & $\begin{array}{l}0.667 \\
1.021 \\
\end{array}$ & \multirow[t]{2}{*}{13.60} & $\begin{array}{l}0.521 \\
0.731 \\
\end{array}$ & \multirow[t]{2}{*}{2100} & $\begin{array}{l}0.700 \\
0.622 \\
\end{array}$ & \multirow[t]{2}{*}{106} & $\begin{array}{l}0228 \\
0.569 \\
\end{array}$ \\
\hline & & & & & 0206 & & 2848 & & 0.665 & & 0.301 \\
\hline
\end{tabular}




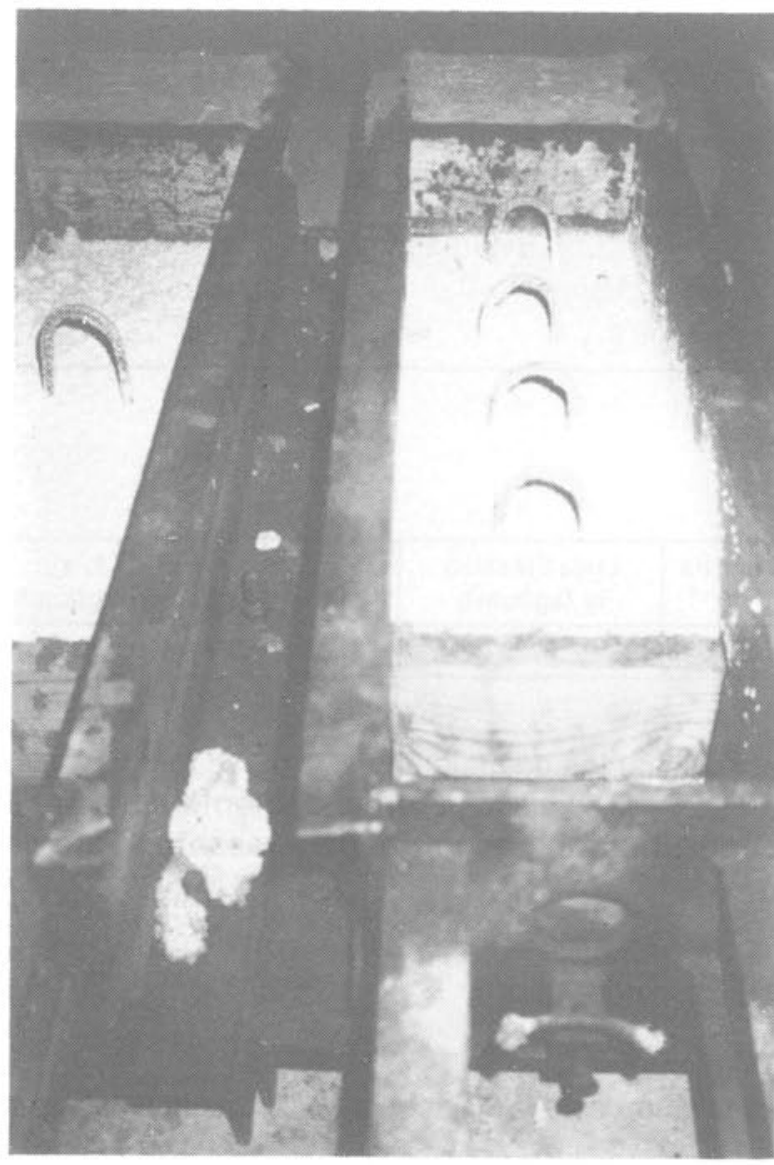

Foto 6.-Serie II-C. Cuantia alta. Pieza tipo 3

\subsubsection{Resumen de resultados de la segunda fase (Serie II-C)}

Este resumen incluye los resultados de los ensayos de las probetas, de acuerdo a las tres cuantias y las cua. tro formas de los estribos cerrados; en él se señala lo siguiente: Tensión tangencial media $(\tau \mathrm{u})$ y deslizamiento medio $(\delta \mathrm{u})$ de los extremos de la junta bajo carga máxima. (Tabla 3.2).

Además se incluyen los resultados de la contribución del hormigón ( $\tau$ uc), como de los coeficientes de eficacia de las armaduras de cosido en la tabla 3.3. a) y b).

\subsection{Ensayos adicionales de juntas sin armadura de cosido}

Se complementaron los ensayos de las series II-A y II$\mathrm{C}$, con ensayos adicionales de juntas sin armaduras de cosido, para determinar la contribución del hormigón ( $\tau$ uc) y el coeficiente $\beta_{1}$. Se respetaron todas las características de las series primitivas referentes a dimensiones, materiales empleados, longitud, etcétera.

Por cada serie II-A y II-C se realizaron 5 probetas para cada una, resumiendo a continuación los resultados medios.

Tabla 3.3.

a) Determinación de la contribución del hormigón.

\begin{tabular}{|c|c|c|c|c|c|}
\hline \multirow{2}{*}{$\begin{array}{c}\text { Serie } \\
\text { Cuantia }\end{array}$} & \multirow{2}{*}{$\begin{array}{c}\text { Cuantia } \\
\delta .10^{-3}\end{array}$} & $\begin{array}{c}\text { fy } \\
\left(\mathrm{kp} / \mathrm{cm}^{2}\right)\end{array}$ & $\begin{array}{c}\delta \mathrm{fy}=\tau \mathrm{us} \\
\left(\mathrm{kp} / \mathrm{cm}^{2}\right)\end{array}$ & $\begin{array}{c}\tau \mathrm{r} \\
\left(\mathrm{kp} / \mathrm{cm}^{2}\right)\end{array}$ & $\begin{array}{c}\text { Contribución Hormigón } \beta 1 \tau \mathrm{uc} \\
\left(\mathrm{kp} / \mathrm{cm}^{2}\right)\end{array}$ \\
\cline { 5 - 6 } & 3,77 & 5.720 & 21,56 & 35,10 & 13,54 \\
II.A & 0,94 & 4.320 & 4,06 & 11,36 & 7,30 \\
II.C-Baja & 1,88 & 4.320 & 8,12 & 15,01 & 6,89 \\
II.C-Alta & 3,77 & 4.320 & 16,29 & 22,86 & 6,57 \\
\hline
\end{tabular}

b) Coeficientes de eficacia de las armaduras de cosido. Coeficientes relativos: $\beta_{1}=1,00$ para el máximo resultado de cada serie que corresponde siempre a las piezas tipo (1)

$\beta_{2}^{*}=\left(\tau \mathrm{uc} \cdot \beta_{1} \tau \mathrm{uc}\right):$ tus

\begin{tabular}{|c|c|c|c|c|c|c|}
\hline \multirow{2}{*}{ Serie } & \multicolumn{3}{c|}{ PIEZAS TIPO } & \multicolumn{2}{c|}{4} \\
\cline { 2 - 7 } Cuantia & \multicolumn{2}{|c|}{2} & \multicolumn{2}{c|}{3} & \multicolumn{2}{c|}{$\tau \mathrm{u}$} \\
$\left(\mathrm{kp} / \mathrm{cm}^{2}\right)$ & $\beta_{2}{ }^{*}$ \\
\cline { 2 - 7 } & $\begin{array}{c}\tau \mathrm{u} \\
\left(\mathrm{kp} / \mathrm{cm}^{2}\right)\end{array}$ & $\beta_{2}{ }^{*}$ & $\begin{array}{c}\tau \mathrm{u} \\
\left(\mathrm{kp} / \mathrm{cm}^{2}\right)\end{array}$ & $\beta_{2}$ & 26,20 & 0,606 \\
\hline II.A & 26,20 & 0,587 & 25,50 & 0,555 & 7,37 & 0,017 \\
II.C-Baja & 8,18 & 0,217 & 10,84 & 0,872 & 11,74 & 0,606 \\
II.C-Media & 11,77 & 0,610 & 15,53 & 1,073 & 11,36 & 0,294 \\
II.C-Alta & 13,60 & 0,432 & 21,08 & 0,891 & - & 0,307 \\
\hline
\end{tabular}

(*) Los valores de $\beta$, $\tau$ uc y de rus se toman de la Tabla 3.3. (a), para cada Serie. 
Tabla 3.4

\begin{tabular}{|c|c|c|c|}
\hline \multicolumn{2}{|c|}{ Longitud de juntas $20 \mathrm{~cm}$} & \multicolumn{2}{|c|}{ Longitud de junta a $40 \mathrm{~cm}$} \\
\hline \multicolumn{2}{|c|}{ Valores medios } & \multicolumn{2}{|c|}{ Valores medios } \\
\hline$\tau \mathrm{u}\left(\mathrm{kgf} / \mathrm{cm}^{2}\right)$ & $\delta \mathrm{u}(\mathrm{mm})$ & $\tau \mathrm{u}\left(\mathrm{kgf} / \mathrm{cm}^{2}\right)$ & $\delta \mathrm{u}(\mathrm{mm})$ \\
\hline 18,78 & 0,059 & 8,6 & 0,134 \\
\hline
\end{tabular}

$\tau \mathrm{u}=$ Tensión cortante máxima

$\delta \mathrm{u}=$ Deslizamiento bajo carga máxima.

\section{CONCLUSIONES Y RECOMENDACIONES}

1. La "Disposición 1" estribos cerrados en el hormigón in situ conduce a los mejores resultados para absorber esfuerzo rasante, si se compara con las otras disposiciones.

Se recomienda:

- En la práctica poner estribos ortogonales al esfuer. zo rasante.

- En las fórmulas de cuantificación del esfuerzo rasante, estipular la disposición de las armaduras.

2. La resistencia de la junta cosida disminuye con el aumento de longitud de ella.

Si se compara:

\begin{tabular}{|c|c|c|}
\cline { 2 - 3 } \multicolumn{1}{c|}{} & $\begin{array}{c}\text { Serie II-A } \\
\text { (Disposición I) }\end{array}$ & $\begin{array}{c}\text { Serie II-C } \\
\text { (Tipo I) }\end{array}$ \\
\hline $\begin{array}{c}\text { Longitud de la junta } \\
\tau \cup\left(\mathrm{kp} / \mathrm{cm}^{2}\right)\end{array}$ & $18 \mathrm{~cm}$ & $38 \mathrm{~cm}$ \\
& 35,1 & 22,9 \\
\hline
\end{tabular}

Al aumentar la longitud de la junta de 18 a $38 \mathrm{~cm}$, se produce una disminución en el esfuerzo rasante de un 35 por ciento.

3. Contribución del hormigón $\left(\beta_{1} \tau\right.$ uc) a esfuerzo rasante. Serie II-A (Disposición I)

$$
\beta_{1} \tau u c=\tau u-\beta_{2} \tau u s
$$

\begin{tabular}{|c|c|c|c|c|}
\hline $\begin{array}{l}\text { Cuantía } \\
\varrho^{\cdot 10^{-3}} \\
\end{array}$ & $\begin{array}{c}\text { Lim. Elástico } \\
\text { fy }\left(\mathbf{k g f t}^{\prime} \mathrm{cm}^{2}\right)\end{array}$ & $\begin{array}{c}\beta_{2} \tau \text { us } \\
\left(\mathrm{kggt} \mathrm{cm}^{2}\right.\end{array}$ & $\begin{array}{c}\tau \mathrm{u} \\
\left(\mathrm{kg}^{f} / \mathrm{cm}^{2}\right)\end{array}$ & $\begin{array}{c}\beta_{1} \tau \mathrm{uc} \\
\left(\mathrm{kgt} / \mathrm{cm}^{2}\right)\end{array}$ \\
\hline 3,77 & 5.720 & 21,56 & 35,10 & 13,54 \\
\hline
\end{tabular}

Para evaluar los coeficientes, $\beta_{1}$ y $\beta_{2}$, se tomó el valor de $\tau \mathrm{uc}=18,8 \mathrm{kgf} / \mathrm{cm}^{2}$, correspondiente al valor de la contribución del hormigón a rasante, junta no armada

determinando que $\beta_{1}=\beta_{2}=0,87$.

La ecuación para la "Disposición 1" queda entonces:

$$
\tau u=0,87(\tau u c+\tau u s)
$$

4. En relación a la serie $\|-C$, se concluye que:

- Las piezas 1 y 3 absorben un $35 \%$ más de esfuerzo rasante que las piezas 2 y 4 .

- La altura del estribo es un factor preponderante en las piezas 1 y 3 , ya que al variar el ancho del estribo no se produjo diferencia sustancial.

5. Contribución del hormigón $\left(\beta_{1} \tau\right.$ uc) en la serie II-C a esfuerzo rasante (piezas tipo 1 y 3 ).

\begin{tabular}{|c|c|c|c|c|c|c|}
\hline & \multicolumn{4}{|c|}{ PIEZA TIPO 1} & \multicolumn{2}{|c|}{ PIEZA TIPO 3} \\
\hline & $\tau \mathrm{u}$ & $\beta_{2}$ tus & $\beta_{1} \tau \mathrm{uc}$ & $\tau u$ & $\beta_{2}$ Tus & $\beta_{1} \tau \mathrm{uc}$ \\
\hline Cuantia & $\left(\mathrm{kgf} / \mathrm{cm}^{2}\right)$ & $\left(\mathrm{kgf} / \mathrm{cm}^{2}\right)$ & $\left(\mathrm{kgf} / \mathrm{cm}^{2}\right)$ & $\left(\mathrm{kgf} / \mathrm{cm}^{2}\right)$ & $\left(\mathrm{kgf} / \mathrm{cm}^{2}\right)$ & $\left(\mathrm{kgf} / \mathrm{cm}^{2}\right)$ \\
\hline $\mathrm{BAJA}=0,94 \cdot 10^{-3}$ & 11,36 & 4,06 & 7,30 & 10,84 & 4,06 & 6,78 \\
\hline MEDIA $=1,88 \cdot 10^{-3}$ & 15,01 & 8,12 & 6,89 & 15,53 & 8,12 & 7,41 \\
\hline$A L T A=3,77 \cdot 10^{-3}$ & 22,86 & 16,29 & 6,57 & 21,08 & 16,29 & 4,79 \\
\hline
\end{tabular}

Dicha contribución se evalúa de acuerdo a la fórmula: 
Para evaluar los coeficientes $\beta_{1}$ y $\beta_{2}$, se tomó el valor de $\tau$ uc $=8,6 \mathrm{kgf} / \mathrm{cm}^{2}$, correspondiente al valor de la contribución del hormigón a rasante de la junta no armada.

- Los valores en las piezas tipo 1, para las tres cuantías: baja, media y alta son idénticos e iguales a:

$$
\beta_{1}=\beta_{2}=0,9
$$

Para evaluar el rasante en las piezas tipo 1 se propone la siguiente fórmula:

$$
\tau u=0,9(\tau u c+\tau u s)
$$

- Los valores en las piezas tipo 3 para las tres cuantías: baja, media y alta son idénticos e iguales a:

$$
\beta_{1}=\beta_{2}=0,88
$$

Se propone en las piezas tipo 3 la siguiente fórmula:

$$
\tau u=0,88(\tau u c+\tau u s)
$$

\section{BIBLIOGRAFIA}

- Hanson, N. W.: Precast prestressed concrete bridgest - Horizontal shear connection. Journal of the Portland Cement Association, Research and Development Laboratories, Vol. 2, May. 1960, pp. 38-58.

- Calavera, J. y otros: Ensayos de corte en la superficie de piezas prefabricadas y hormigones vertidos in situ. Hormigón y Acẹo, n. 111 y 120, 1976.

- Dardare, J.: Estude des contraintes de cisaillementglissement dans les elements composites soumis a la flexion. Centre d'Estudes et de Recherches de l'Industrie du Beton Manifacture. Monografhie n..$^{\circ}$, junio 1970.

- Saeman, J. C. y otros: Horizontal shear connection between beams and cast in place slabs. Journal of the Ame. rican Concrete Institute, Nov. 1964, Proceedings V. 61, n. ${ }^{\circ} 11$.

- Mattock, A. H.: Shear transfer in reinforced concrete recent research. Prestressed Cement Association, Journal, March-April 1972.

- Mattock, A. H.: Shear transfer in reinforced concrete. American Concrete Institute. Journal, Vol. 66, n. ${ }^{\circ}$ 2, Feb. 1969.

\section{publicación del ietcc / CSIC}

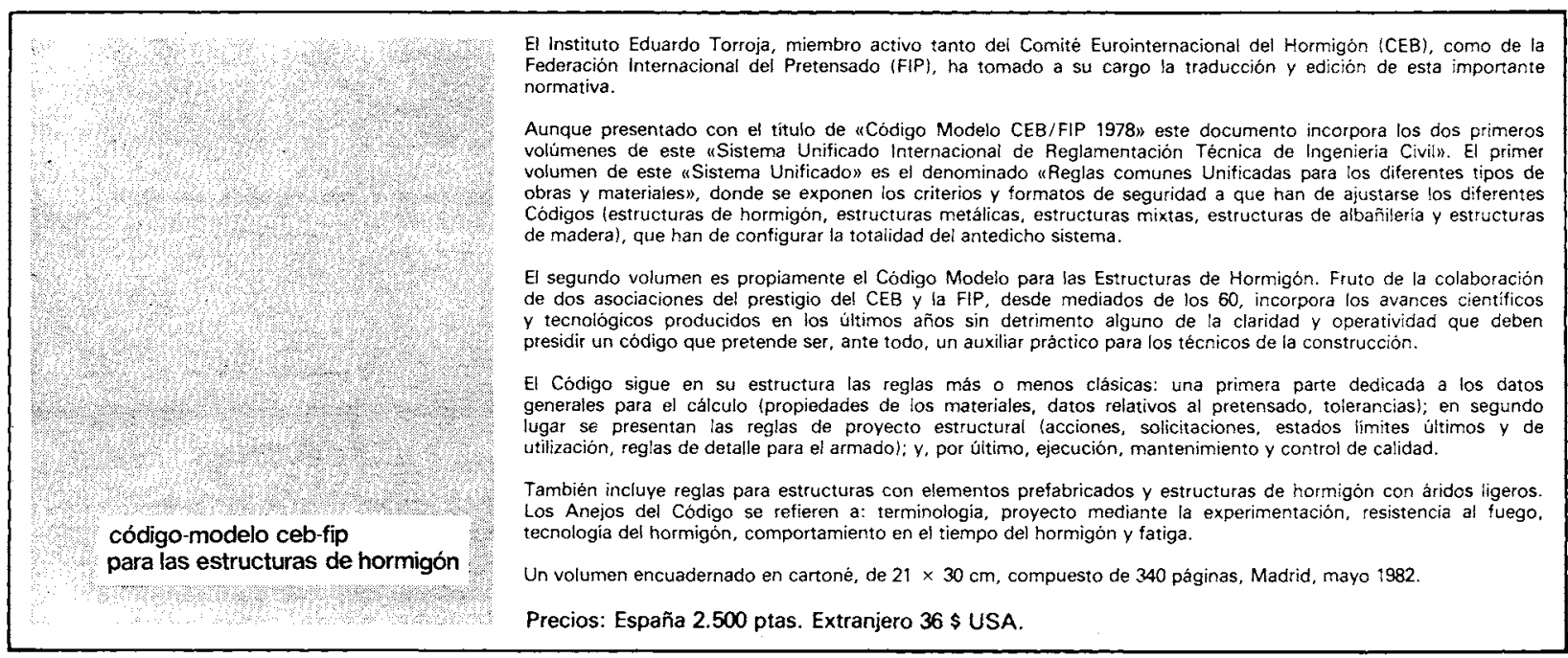

